\title{
$p$-adic measures associated with zeta values and $p$-adic log multiple gamma functions
}

\author{
Tomokazu Kashio*
}

October 19, 2018

\begin{abstract}
We study a relation between two refinements of the rank one abelian Gross-Stark conjecture: For a suitable abelian extension $H / F$ of number fields, a Gross-Stark unit is defined as a $p$-unit of $H$ satisfying some proporties. Let $\tau \in \operatorname{Gal}(H / F)$. Yoshida and the author constructed the symbol $Y_{p}(\tau)$ by using $p$-adic log multiple gamma functions, and conjectured that the $\log _{p}$ of a Gross-Stark unit can be expressed by $Y_{p}(\tau)$. Dasgupta constructed the symbol $u_{T}(\tau)$ by using the $p$-adic multiplicative integration, and conjectured that a Gross-Stark unit can be expressed by $u_{T}(\tau)$. In this paper, we give an explicit relation between $Y_{p}(\tau)$ and $u_{T}(\tau)$.
\end{abstract}

\section{Introduction}

Let $F$ be a totally real field, $K$ a CM-field which is abelian over $F, S$ a finite set of places of $F$. We assume that

- $S$ contains all infinite places of $F$, all places of $F$ lying above a rational prime $p$, and all ramified places in $K / F$.

- Let $\mathfrak{p}$ be the prime ideal corresponding to the $p$-adic topology on $F$. (Hence $\mathfrak{p} \in S$.) Then $\mathfrak{p}$ splits completely in $K / F$.

For $\tau \in \operatorname{Gal}(K / F)$, we consider the partial zeta function

$$
\zeta_{S}(s, \tau):=\sum_{\left(\frac{K / F}{\mathfrak{a}}\right)=\tau,(\mathfrak{a}, S)=1} N \mathfrak{a}^{-s} .
$$

Here $\mathfrak{a}$ runs over all integral ideals of $F$, relatively prime to any finite places in $S$, whose image under the Artin symbol $\left(\frac{K / F}{*}\right)$ is equal to $\tau$. The series converges for $\operatorname{Re}(s)>1$, has a meromorphic continuation to the whole $s$-plane, and is analytic at $s=0$. Moreover, under our assumption, we see that

2010 Mathematics subject classification(s). 11R27, 11R37, 11R42, 11R80, 11S40, 11S80, 33B15.

Key words and phrases. the Gross-Stark conjecture, multiple gamma functions, $p$-adic measures.

*Tokyo University of Science, kashio_tomokazu@ma.noda.tus.ac.jp 
- There exists the $p$-adic interpolation function $\zeta_{p, S}(s, \tau)$ of $\zeta_{S}(s, \tau)$.

- $\operatorname{ord}_{s=0} \zeta_{S}(s, \tau), \operatorname{ord}_{s=0} \zeta_{p, S}(s, \tau) \geq 1$.

- There exist a natural number $W$, a $\mathfrak{p}$-unit $u$ of $K$, which satisfy

$$
\log \left|u^{\tau}\right|_{\mathfrak{P}}=-W \zeta_{S}^{\prime}(0, \tau) \quad(\tau \in \operatorname{Gal}(K / F)) .
$$

Here $\mathfrak{P}$ denotes the prime ideal corresponding to the $p$-adic topology on $K,|x|_{\mathfrak{P}}:=$ $N \mathfrak{P}^{-\operatorname{ord}_{\mathfrak{P}} x}$.

Gross conjectured the following $p$-adic analogue of the rank 1 abelian Stark conjecture:

Conjecture 1 ([Gr, Conjecture 3.13]). Let $u$ be a $\mathfrak{p}$-unit characterized by (1) up to roots of unity. Then we have

$$
\log _{p} N_{K_{\mathfrak{P}} / \mathbb{Q}_{p}}\left(u^{\tau}\right)=-W \zeta_{p, S}(0, \tau) .
$$

Dasgupta-Darmon-Pollack [DDP] proved a large part of Conjecture1. Yoshida and the author, and independently Dasgupta formulated refinements of Conjecture1: Let $\mathfrak{f}$ be an integral ideal of a totally real field $F$ satisfying $\mathfrak{p} \nmid \mathfrak{f}, H_{\mathfrak{f}}$ the narrow ray class field modulo $\mathfrak{f}, H$ the maximal subfield of $H_{\mathfrak{f}}$ where $\mathfrak{p}$ splits completely. Yoshida and the author [KY1] essentially constructed the invariant $Y_{p}(\tau)$ (Definition 6) for $\tau \in \operatorname{Gal}(H / F)$ by using $p$-adic $\log$ multiple gamma functions. Then [KY1, Conjecture $\left.\mathrm{A}^{\prime}\right]$ states that $\log _{p} u^{\tau}$ (without $N_{K_{\mathfrak{Y}} / \mathbb{Q}_{p}}$ ) can be expressed by $Y_{p}(\tau)$. On the other hand, Dasgupta constructed the invariant $u_{T}\left(\mathfrak{b}, \mathcal{D}_{\mathfrak{f}}\right)$ (Definition 12 (iv)) by using the multiplicative integration for $p$ adic measures associated with Shintani's multiple zeta functions. Then [Da, Conjecture 3.21] states that a modified version of $u^{\tau}$ can be expressed by $u_{T}\left(\mathfrak{b}, \mathcal{D}_{\mathfrak{f}}\right)$. In [Ka3, Remark $2]$, the author announced the following relation between these refinements.

Theorem (Theorem 11). Let $\eta$ be a "good" prime ideal in the sense of Definition 11. We put $T:=\{\eta\}$. Then we have

$$
\log _{p}\left(u_{\eta}\left(\mathfrak{b}, \mathcal{D}_{\mathfrak{f}}\right)\right)=-Y_{p}\left(\left(\frac{H / F}{\mathfrak{b}}\right)\right)+N \eta Y_{p}\left(\left(\frac{H / F}{\mathfrak{b} \eta^{-1}}\right)\right) .
$$

In particular, we see that two refinements are consistent (roughly speaking, [Da, Conjecture 3.21] is a further refinement of [KY1, Conjecture $\left.\mathrm{A}^{\prime}\right]$ by $\left.\operatorname{ker} \log _{p}\right)$. The aim of this paper is to prove this Theorem.

Let us explain the outline of this paper. In $\S 2$, we introduce Shintani's technique of cone decompositions. We obtain a suitable fundamental domain of $F \otimes \mathbb{R}_{+} / E_{\mathfrak{f},+}$, where $F \otimes \mathbb{R}_{+}$denotes the totally positive part of $F \otimes \mathbb{R}, E_{\mathfrak{f},+}$ is a subgroup of the group of all totally positive units. We need such fundamental domains in order to construct both of the invariants $Y_{p}, u_{T}$. In $\S 3$, we recall the definition and some properties of $Y_{p}$, which is essentially defined in [KY1 and slightly modified in [Ka3]. The classical or $p$-adic log multiple gamma function is defined as the derivative values at $a=0$ of the classical or $p$-adic Barnes' multiple zeta function, respectively. Then the invariant $Y_{p}(\tau, \iota)$ is defined in Definition 6, as a finite sum of the "difference" of $p$-adic log multiple gamma functions and classical log multiple gamma functions. Conjecture 2 predicts exact values 
of $Y_{p}(\tau, \iota)$. In $\S 4$, we also recall some results in [Da]. Dasgupta introduced $p$-adic measures $\nu_{T}\left(\mathfrak{b}, \mathcal{D}_{\mathfrak{f}}\right)$ associated with special values of Shintani's multiple zeta functions, and defined $u_{T}\left(\mathfrak{b}, \mathcal{D}_{\mathfrak{f}}\right)$ as the multiplicative integration $\mathcal{*}_{\mathrm{O}} x d \nu_{\eta}\left(\mathfrak{b}, \mathcal{D}_{\mathfrak{f}}, x\right)$ with certain correction terms. Dasgupta formulated a conjecture (Conjecture 3) on properties of $u_{T}\left(\mathfrak{b}, \mathcal{D}_{\mathfrak{f}}\right)$. In $\S 5$, we state and prove the main result (Theorem 1) which gives an explicit relation between $Y_{p}(\tau, \mathrm{id})$ and $\log _{p}\left(u_{\eta}\left(\mathfrak{b}, \mathcal{D}_{\mathfrak{f}}\right)\right)$. Then we will see that Conjectures 2 , 3 are consistent in the sense of Corollary 1. The key observation is Lemma 3. Dasgupta's $p$-adic measure $\nu_{\eta}\left(\mathfrak{b}, \mathcal{D}_{\mathfrak{f}}\right)$ is originally associated with Shintani's multiple zeta functions. By this Lemma, we can relate $\nu_{\eta}\left(\mathfrak{b}, \mathcal{D}_{\mathfrak{f}}\right)$ to Barnes' multiple zeta functions and $p$-adic analogues as in Lemma 4.

\section{Shintani domains}

Let $F$ be a totally real field of degree $n, \mathcal{O}_{F}$ the ring of integers of $F, \mathfrak{f}$ an integral ideal of $F$. We denote by $F_{+}$the set of all totally positive elements in $F$ and put $\mathcal{O}_{F,+}:=\mathcal{O}_{F} \cap F_{+}$, $E_{+}:=\mathcal{O}_{F}^{\times} \cap F_{+}$. We consider subgroups of $E_{+}$of the following form:

$$
E_{\mathfrak{f},+}:=\left\{\epsilon \in E_{+} \mid \epsilon \equiv 1 \bmod \mathfrak{f}\right\} .
$$

We identify

$$
F \otimes \mathbb{R}=\mathbb{R}^{n}, \quad \sum_{i=1}^{k} a_{i} \otimes b_{i} \mapsto\left(\sum_{i=1}^{k} \iota\left(a_{i}\right) b_{i}\right)_{\iota \in \operatorname{Hom}(F, \mathbb{R})},
$$

where $\operatorname{Hom}(F, \mathbb{R})$ denotes the set of all real embeddings of $F$. In particular, the totally positive part

$$
F \otimes \mathbb{R}_{+}:=\mathbb{R}_{+}^{n}
$$

has a meaning. On the right-hand side, $\mathbb{R}_{+}$denotes the set of all positive real numbers. Let $v_{1}, \ldots, v_{r} \in \mathcal{O}_{F}$ be linearly independent. Then we define the cone with basis $\boldsymbol{v}:=$ $\left(v_{1}, \ldots, v_{r}\right)$ as

$$
C(\boldsymbol{v}):=\left\{\boldsymbol{t}^{t} \boldsymbol{v} \in F \otimes \mathbb{R} \mid \boldsymbol{t} \in \mathbb{R}_{+}^{r}\right\} .
$$

Here we $\boldsymbol{t}^{t} \boldsymbol{v}$ denotes the inner product.

Definition 1. (i) We call a subset $D \subset F \otimes \mathbb{R}_{+}$is a Shintani set if it can be expressed as a finite disjoint union of cones:

$$
D=\coprod_{i \in J} C\left(\boldsymbol{v}_{j}\right) \quad\left(|J|<\infty, \boldsymbol{v}_{j} \in \mathcal{O}_{F,+}^{r(j)}, r(j) \in \mathbb{N}\right) .
$$

(ii) We consider the natural action $E_{\mathfrak{f},+} \curvearrowright F \otimes \mathbb{R}_{+}, u(a \otimes b):=(u a) \otimes b$. We call $a$ Shintani set $D$ a Shintani domain $\bmod E_{\mathfrak{f},+}$ if it is a fundamental domain of $F \otimes$ $\mathbb{R}_{+} / E_{\mathfrak{f},+}$ :

$$
F \otimes \mathbb{R}_{+}=\coprod_{\epsilon \in E_{\mathfrak{f},+}} \epsilon D
$$

When $\mathfrak{f}=(1)$, we write $\bmod E_{+}$instead of $\bmod E_{(1),+}$.

Shintani [Sh, Proposition 4] showed that there always exists a Shintani domain. 


\section{$3 \quad p$-adic log multiple gamma functions}

We recall the definition and some properties of the symbol $Y_{p}$ defined in [KY1], [Ka3]. We denote by $\mathbb{R}_{+}$the set of all positive real numbers.

Definition 2. Let $z \in \mathbb{R}_{+}, \boldsymbol{v} \in \mathbb{R}_{+}^{r}$. Barnes' multiple zeta function is defined as

$$
\zeta(s, \boldsymbol{v}, z):=\sum_{\boldsymbol{m} \in \mathbb{Z}_{\geq 0}^{r}}\left(z+\boldsymbol{m}^{t} \boldsymbol{v}\right)^{-s} .
$$

This series converges for $\operatorname{Re}(s)>r$, has a meromorphic continuation to the whole s-plane, and is analytic at $s=0$. Then Barnes' multiple gamma function is defined as

$$
\Gamma(z, \boldsymbol{v}):=\exp \left(\left.\frac{\partial}{\partial s} \zeta(s, \boldsymbol{v}, z)\right|_{s=0}\right) .
$$

Note that this definition is modified from that given by Barnes. For the proof and details, see [Yo, Chap I, §1]. Throughout this paper, we regard each number field as a subfield of $\overline{\mathbb{Q}}$, and fix two embeddings $\overline{\mathbb{Q}} \hookrightarrow \mathbb{C}, \overline{\mathbb{Q}} \hookrightarrow \mathbb{C}_{p}$. Here $\mathbb{C}_{p}$ denotes the $p$-adic completion of the algebraic closure of $\mathbb{Q}_{p}$. We denote by $\mu_{(p)}$ the group of all roots of unity of prime-to- $p$ order. Let $\operatorname{ord}_{p}: \mathbb{C}_{p}^{\times} \rightarrow \mathbb{Q}, \theta_{p}: \mathbb{C}_{p}^{\times} \rightarrow \mu_{(p)}$ be unique group homomorphisms satisfying

$$
\left|p^{-\operatorname{ord}_{p}(z)} \theta_{p}(z)^{-1} z\right|_{p}<1 \quad\left(z \in \mathbb{C}_{p}^{\times}\right)
$$

Definition 3. Let $z \in \overline{\mathbb{Q}}, \boldsymbol{v} \in\left(\overline{\mathbb{Q}}^{\times}\right)^{r}$. We assume that

$$
\begin{aligned}
& z \in \mathbb{R}_{+}, \boldsymbol{v} \in \mathbb{R}_{+}^{r} \text { via the embedding } \overline{\mathbb{Q}} \hookrightarrow \mathbb{C}, \\
& \operatorname{ord}_{p}(z)<\operatorname{ord}_{p}\left(v_{1}\right), \ldots, \operatorname{ord}_{p}\left(v_{r}\right) \text { via the embedding } \overline{\mathbb{Q}} \hookrightarrow \mathbb{C}_{p} .
\end{aligned}
$$

Then we denote by $\zeta_{p}(s, \boldsymbol{v}, z)\left(s \in \mathbb{Z}_{p}-\{1,2, \ldots, r\}\right)$ the p-adic multiple zeta function characterized by

$$
\zeta_{p}(-m, \boldsymbol{v}, z)=p^{-\operatorname{ord}_{p}(z) m} \theta_{p}(z)^{-m} \zeta(-m, \boldsymbol{v}, z) \quad\left(m \in \mathbb{Z}_{\geq 0}\right) .
$$

We define the p-adic log multiple gamma function as

$$
L \Gamma_{p}(z, \boldsymbol{v}):=\left.\frac{\partial}{\partial s} \zeta_{p}(s, \boldsymbol{v}, z)\right|_{s=0} .
$$

The construction of $\zeta_{p}(s, \boldsymbol{v}, z)$ is due to Cassou-Noguès [CN1]. The author defined and studied $L \Gamma_{p}(z, \boldsymbol{v})$ in [Ka1]. See [Ka3, $\left.\S 2\right]$ for a short survey.

Definition 4. Let $F$ be a totally real field, $\mathfrak{f}$ an integral ideal, $D=\coprod_{j \in J} C\left(\boldsymbol{v}_{j}\right)\left(\boldsymbol{v}_{j} \in \mathcal{O}_{F,+}^{r(j)}\right)$ a Shintani domain $\bmod E_{+}$. We denote by $\operatorname{Hom}(F, \mathbb{R})\left(\operatorname{resp} \operatorname{Hom}\left(F, \mathbb{C}_{p}\right)\right)$ the set of all embeddings of $F$ into $\mathbb{R}$ (resp. $\mathbb{C}_{p}$ ). Since we fixed embeddings $\overline{\mathbb{Q}} \hookrightarrow \mathbb{C}, \overline{\mathbb{Q}} \hookrightarrow \mathbb{C}_{p}$, we may identify

$$
\operatorname{Hom}(F, \mathbb{R})=\operatorname{Hom}\left(F, \mathbb{C}_{p}\right)
$$


(i) We denote by $C_{\mathfrak{f}}$ the narrow ideal class group modulo $\mathfrak{f}$, by $H_{\mathfrak{f}}$ the narrow ray class field modulo f. In particular, the Artin map induces

$$
C_{\mathfrak{f}} \cong \operatorname{Gal}\left(H_{\mathfrak{f}} / F\right) \text {. }
$$

(ii) Let $\pi: C_{\mathfrak{f}} \rightarrow C_{(1)}$ be the natural projection. For each $c \in C_{\mathfrak{f}}$, we take an integral ideal $\mathfrak{a}_{c}$ satisfying

$$
\mathfrak{a}_{c} \mathfrak{f} \in \pi(c)
$$

(iii) For $c \in C_{\mathfrak{f}}, \boldsymbol{v} \in \mathcal{O}_{F}^{r}$, we put

$$
R(c, \boldsymbol{v}):=R\left(c, \boldsymbol{v}, \mathfrak{a}_{c}\right):=\left\{\boldsymbol{x} \in(\mathbb{Q} \cap(0,1])^{r} \mid \mathcal{O}_{F} \supset\left(\boldsymbol{x}^{t} \boldsymbol{v}\right) \mathfrak{a}_{c} \mathfrak{f} \in c\right\}
$$

(iv) For $c \in C_{\mathfrak{f}}, \iota \in \operatorname{Hom}(F, \mathbb{R})$, we define

$$
G(c, \iota):=G\left(c, \iota, D, \mathfrak{a}_{c}\right):=\sum_{j \in J} \sum_{\boldsymbol{x} \in R\left(c, \boldsymbol{v}_{j}\right)} \log \Gamma\left(\iota\left(\boldsymbol{x}^{t} \boldsymbol{v}_{j}\right), \iota\left(\boldsymbol{v}_{j}\right)\right) .
$$

$(\mathrm{v})$ For $\iota \in \operatorname{Hom}(F, \mathbb{R})\left(=\operatorname{Hom}\left(F, \mathbb{C}_{p}\right)\right)$, we put

$$
\mathfrak{p}_{\iota}:=\left\{\left.z \in \mathcal{O}_{F}|| \iota(z)\right|_{p}<1\right\}
$$

Note that the prime ideal $\iota\left(\mathfrak{p}_{\iota}\right)$ corresponds to the $p$-adic topology on $\iota(F) \subset \mathbb{C}_{p}$.

(vi) Assume that $\mathfrak{p}_{\iota} \mid \mathfrak{f}$. For $c \in C_{\mathfrak{f}}, \iota \in \operatorname{Hom}(F, \mathbb{R})$, we define

$$
G_{p}(c, \iota):=G_{p}\left(c, \iota, D, \mathfrak{a}_{c}\right):=\sum_{j \in J} \sum_{\boldsymbol{x} \in R\left(c, \boldsymbol{v}_{j}\right)} L \Gamma_{p}\left(\iota\left(\boldsymbol{x}^{t} \boldsymbol{v}_{j}\right), \iota\left(\boldsymbol{v}_{j}\right)\right)
$$

Note that $\left(\iota\left(\boldsymbol{x}^{t} \boldsymbol{v}_{j}\right), \iota\left(\boldsymbol{v}_{j}\right)\right)$ satisfies the assumption (3) whenever $\mathfrak{p}_{\iota} \mid \mathfrak{f}, \boldsymbol{x} \in R\left(c, \boldsymbol{v}_{j}\right)$. The following map [ $]_{p}$ is well-defined by [KY1, Lemma 5.1].

Definition 5. We denote by $\overline{\mathbb{Q}} \log _{p} \overline{\mathbb{Q}}^{\times}\left(\right.$resp. $\left.\overline{\mathbb{Q}} \log \overline{\mathbb{Q}}^{\times}\right)$the $\overline{\mathbb{Q}}$-subspace of $\mathbb{C}_{p}$ (resp. $\left.\mathbb{C}\right)$ generated by $\log _{p} b($ resp. $\pi, \log b)$ with $b \in \overline{\mathbb{Q}}^{\times}$. We define a $\overline{\mathbb{Q}}$-linear map []$_{p}$ by

$$
[]_{p}: \overline{\mathbb{Q}} \log \overline{\mathbb{Q}}^{\times} \rightarrow \overline{\mathbb{Q}} \log _{p} \overline{\mathbb{Q}}^{\times}, \quad a \log b \mapsto a \log _{p} b, \quad a \pi \mapsto 0 \quad(a, b \in \overline{\mathbb{Q}}, b \neq 0) .
$$

Lemma 1. Let $H$ be an intermediate field of $H_{\mathfrak{f}} / F, \mathfrak{q}$ a prime ideal of $F$, relatively prime to $\mathfrak{f}$, splitting completely in $H / F$. Then we have

$$
\sum_{c \in C_{\mathfrak{f q}},\left.\operatorname{Art}(\bar{c})\right|_{H}=\tau} G(c, \iota) \in \overline{\mathbb{Q}} \log \overline{\mathbb{Q}}^{\times} \quad(\tau \in \operatorname{Gal}(H / F)) .
$$

Here c runs over all ideal classes whose images under the composite map $C_{\mathfrak{f q}} \rightarrow C_{\mathfrak{f}} \rightarrow$ $\operatorname{Gal}\left(H_{\mathfrak{f}} / F\right) \rightarrow \operatorname{Gal}(H / F)$ is equal to $\tau$. 
Proof. We put $W(c, \iota):=W(\iota(c))$ in [KY1, (4.3)], $V(c, \iota):=V(\iota(c))$ in [KY1, (1.6)], and $X(c, \iota):=G(c, \iota)+W(c, \iota)+V(c, \iota)$. Here we consider the ideal class group $C_{\iota(\mathfrak{f})}$ of $\iota(F)$ modulo $\iota(\mathfrak{f})$. Then $\iota(c)$ denotes the image of $c \in C_{\mathfrak{f}}$ in $C_{\iota(\mathfrak{f})}$ under the natural map. By the definition [KY1, (4.3)] and [KY2, Appendix I, Theorem], we have $W(c, \iota), V(c, \iota) \in$ $\overline{\mathbb{Q}} \log \overline{\mathbb{Q}}^{\times}$. Moreover [KY1, Lemma 5.5] states that

$$
\sum_{c \in C_{\mathfrak{f} \mathfrak{q}}} \chi_{\mathfrak{q}}(c) X(c, \iota) \in \overline{\mathbb{Q}} \log \overline{\mathbb{Q}}^{\times} \quad\left(\chi \in \hat{C}_{\mathfrak{f}}, \chi([\mathfrak{q}])=1\right) .
$$

Here $\chi_{\mathfrak{q}},[\mathfrak{q}]$ denote the composite map $C_{\mathfrak{f q}} \rightarrow C_{\mathfrak{f}} \stackrel{\chi}{\rightarrow} \mathbb{C}^{\times}$, the ideal class $\in C_{\mathfrak{f}}$ of $\mathfrak{q}$, respectively. Therefore, when $H$ is the fixed subfield under $\left(\frac{H_{\mathfrak{f}} / F}{\mathfrak{q}}\right)$, it follows from the orthogonality of characters. The general case follows from this case immediately.

Definition 6. Let $H$ be an intermediate field of $H_{\mathfrak{f}} / F$. Assume that $\mathfrak{p}_{\iota} \nmid \mathfrak{f}$ and that $\mathfrak{p}_{\iota}$ splits completely in $H / F$. Then we define

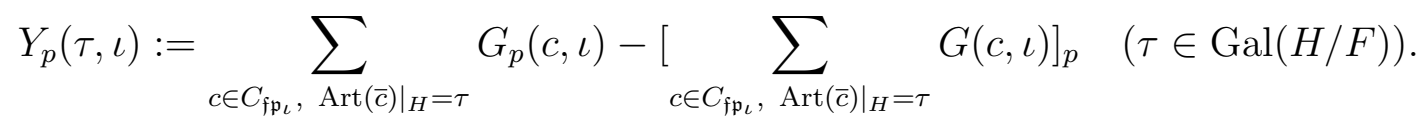

When $\iota=\mathrm{id}$, we drop the symbol $\iota: Y_{p}(\tau):=Y_{p}(\tau, \mathrm{id})$.

By [KY1, Proposition 5.6] (and the orthogonality of characters), we see that $Y_{p}(\tau, \iota)$ depends only on $H, \mathfrak{f}, \tau, \iota$, not on $D, \mathfrak{a}_{c}$ 's. We formulated a conjecture [KY1, Conjecture $\mathrm{A}^{\prime}$ ], which is equivalent to the following Conjecture 2 by [Ka3, Proposition 6-(ii)].

Conjecture 2. Let $H_{\mathfrak{f}} / H / F$ be as above: we assume that

$\mathfrak{p}_{\iota}$ does not divide $\mathfrak{f}$, splits completely in $H / F$.

We take a lift $\tilde{\iota}: H \rightarrow \mathbb{C}_{p}$ of $\iota: F \rightarrow \mathbb{C}_{p}$ and put $\mathfrak{p}_{H, \tilde{\tau}}:=\left\{\left.z \in \mathcal{O}_{H}|| \tilde{\iota}(z)\right|_{p}<1\right\}$. Let $\alpha_{H, \tilde{\iota}}$ be a generator of the principal ideal $\mathfrak{p}_{H, \tilde{\iota}}^{h_{H}}$, where $h_{H}$ denotes the class number. Then we have

$$
Y_{p}(\tau, \iota)=\frac{-1}{h_{H}} \sum_{c \in C_{\mathfrak{f}}} \zeta\left(0, c^{-1}\right) \log _{p} \tilde{\iota}\left(\alpha_{H, \tilde{\iota}}^{\tau \operatorname{Art}(c)}\right)
$$

Remark 1. Roughly speaking, the above conjecture states a relation between the ratios [ p-adic multiple gamma functions: multiple gamma functions ] and Stark units associated with the finite place $\mathfrak{p}_{\iota}$. We also studied a relation between the same ratios and Stark units associated with real places in [Ka3]. We found a more significant relation between the ratios [ $p$-adic gamma function : gamma function] and cyclotomic units in [Ka2].

We rewrite the definition of $Y_{p}$ for later use.

Definition 7. Let $R$ be a subset of $F_{+}$. We assume that $R$ can be expressed in the following form:

$$
R=\coprod_{i=1}^{k}\left\{\left(\boldsymbol{x}_{i}+\boldsymbol{m}\right)^{t} \boldsymbol{v}_{i} \mid \boldsymbol{m} \in \mathbb{Z}_{\geq 0}^{r_{i}}\right\} \quad\left(\boldsymbol{x}_{i} \in \mathbb{Q}_{+}^{r_{i}}, \boldsymbol{v}_{i} \in F_{+}^{r_{i}}\right) .
$$


(i) We define

$$
\begin{aligned}
& \zeta_{\iota}(s, R):=\sum_{z \in R} \iota(z)^{-s}:=\sum_{i=1}^{k} \zeta\left(s, \iota\left(\boldsymbol{v}_{i}\right), \iota\left(\boldsymbol{x}_{i}{ }^{t} \boldsymbol{v}_{i}\right)\right) \\
& L \Gamma_{\iota}(R):=\left.\frac{\partial}{\partial s} \zeta_{\iota}(s, R)\right|_{s=0}=\sum_{i=1}^{k} \log \Gamma\left(\iota\left(\boldsymbol{x}_{i}{ }^{t} \boldsymbol{v}_{i}\right), \iota\left(\boldsymbol{v}_{i}\right)\right) .
\end{aligned}
$$

(ii) Additionally we assume that each $\left(\iota\left(\boldsymbol{x}_{i}{ }^{t} \boldsymbol{v}_{i}\right), \iota\left(\boldsymbol{v}_{i}\right)\right)$ satisfies (3). Then there exists the p-adic interpolation function

$$
\zeta_{\iota, p}(s, R):=\sum_{i=1}^{k} \zeta_{p}\left(s, \iota\left(\boldsymbol{v}_{i}\right), \iota\left(\boldsymbol{x}_{i}{ }^{t} \boldsymbol{v}_{i}\right)\right)
$$

of $\zeta_{\iota}(s, R)$. We define

$$
L \Gamma_{\iota, p}(R):=\left.\frac{\partial}{\partial s} \zeta_{\iota, p}(s, R)\right|_{s=0}=\sum_{i=1}^{k} L \Gamma_{p}\left(\iota\left(\boldsymbol{x}_{i}{ }^{t} \boldsymbol{v}_{i}\right), \iota\left(\boldsymbol{v}_{i}\right)\right)
$$

When $\iota=\mathrm{id}$, we drop the symbol $\iota$.

It follows that, for any Shintani domain $D \bmod E_{+}$and for any integral ideals $\mathfrak{a}_{c}$ satisfying $\mathfrak{a}_{c} \mathfrak{f} \in \pi(c)$, we have

$$
Y_{p}(\tau, \iota)=\sum_{c \in C_{\mathfrak{f} \mathfrak{p}},} L \Gamma_{\iota, p}\left(R_{c}\right)-\left[\sum_{c \in C_{\mathfrak{f} \mathfrak{p}_{\iota}},\left.\operatorname{Art}(\bar{c})\right|_{H}=\tau} L \Gamma_{\iota}\left(R_{c}\right)\right]_{p},
$$

where we put $R_{c}:=\left\{z \in D \mid \mathcal{O}_{F} \supset z \mathfrak{a}_{c} \mathfrak{f p}_{\iota} \in c\right\}$. We will use the following properties of the classical or $p$-adic multiple gamma functions in the proof of Theorem 1 .

Proposition 1. (i) Let $R$ be as in Definition 7-(i), $\alpha \in F_{+}$. Then we have

$$
L \Gamma_{\iota}(R)-L \Gamma_{\iota}(\alpha R)=\zeta_{\iota}(0, R) \log \iota(\alpha)
$$

(ii) Let $R$ be as in Definition 7 (ii), $\alpha \in F_{+}$. Then we have

$$
L \Gamma_{\iota, p}(R)-L \Gamma_{\iota, p}(\alpha R)=\zeta_{\iota}(0, R) \log _{p} \iota(\alpha) .
$$

Proof. The assertions follow from $\zeta_{\iota}(s, \alpha R)=\iota(\alpha)^{-s} \zeta_{\iota}(s, R)$ immediately.

We also recall Shintani's multiple zeta functions in [Sh, (1.1)] which we need in subsequent sections.

Definition 8. (i) Let $A=\left(a_{i j}\right)$ be an $(l \times r)$-matrix with $a_{i j} \in \mathbb{R}_{+}, \boldsymbol{x} \in \mathbb{R}_{+}^{r}$, $\boldsymbol{\chi}=$ $\left(\chi_{1}, \ldots, \chi_{r}\right) \in\left(\mathbb{C}^{\times}\right)^{r}$ with $\left|\chi_{i}\right| \leq 1$. Then Shintani's multiple zeta function is defined as

$$
\zeta(s, A, \boldsymbol{x}, \boldsymbol{\chi}):=\sum_{\left(m_{1}, \ldots, m_{r}\right) \in \mathbb{Z}_{\geq 0}^{r}}\left(\prod_{j=1}^{r} \chi_{j}^{m_{j}}\right)\left(\prod_{i=1}^{l}\left(\sum_{j=1}^{r} a_{i j}\left(m_{j}+x_{j}\right)\right)\right)^{-s} .
$$

This series converges for $\operatorname{Re}(s)>\frac{r}{l}$, has a meromorphic continuation to the whole s-plane, is analytic at $s=0$. 
(ii) Let $\boldsymbol{x}, \chi$ be as in (i). For $\boldsymbol{v}=\left(v_{1}, \ldots, v_{r}\right) \in F_{+}^{r}$, we consider two kinds of Shintani's multiple zeta functions:

(a) Shintani's multiple zeta function with $l=1$ :

$$
\zeta(s, \boldsymbol{v}, \boldsymbol{x}, \boldsymbol{\chi})=\sum_{\left(m_{1}, \ldots, m_{r}\right) \in \mathbb{Z}_{\geq 0}^{r}}\left(\prod_{j=1}^{r} \chi_{j}^{m_{j}}\right)\left(\sum_{j=1}^{r} v_{j}\left(m_{j}+x_{j}\right)\right)^{-s} .
$$

Here we consider $v_{i} \in F_{+} \stackrel{\text { id }}{\hookrightarrow} \mathbb{R}_{+}$.

(b) Let $A$ be the $(n \times r)$-matrix whose raw vectors are $\iota\left(\boldsymbol{v}_{i}\right)(\iota \in \operatorname{Hom}(F, \mathbb{R}), n:=$ $[F: \mathbb{Q}])$. Then we put

$$
\zeta_{N}(s, \boldsymbol{v}, \boldsymbol{x}, \boldsymbol{\chi}):=\zeta(s, A, \boldsymbol{x}, \boldsymbol{\chi})=\sum_{\left(m_{1}, \ldots, m_{r}\right) \in \mathbb{Z}_{\geq 0}^{r}}\left(\prod_{j=1}^{r} \chi_{j}^{m_{j}}\right) N\left(\sum_{j=1}^{r} v_{j}\left(m_{j}+x_{j}\right)\right)^{-s}
$$

(iii) Let $R$ be as in Definition 7f(i). We define

$$
\zeta_{N}(s, R):=\sum_{z \in R} N z^{-s}:=\sum_{i=1}^{k} \zeta_{N}\left(s, \boldsymbol{v}_{i}, \boldsymbol{x}_{i},(1, \ldots, 1)\right)
$$

\section{4 -adic measures associated with zeta values}

We consider the following two kinds of integration.

Definition 9. Let $K$ be a finite extension of $\mathbb{Q}_{p}, O$ the ring of integers of $K$, $P$ the maximal ideal of $O$.

(i) We say $\nu$ is a p-adic measure on $O$ if for each open compact subset $U \subset O$, it takes the value $\nu(U) \in K$ satisfying

(a) $\nu\left(U \coprod U^{\prime}\right)=\nu(U)+\nu\left(U^{\prime}\right)$ for disjoint open compact subsets $U, U^{\prime}$.

(b) $|\nu(U)|_{p}$ 's are bounded.

We say a $p$-adic measure $\nu$ is a $\mathbb{Z}$-valued measure if $\nu(U) \in \mathbb{Z}$.

(ii) Let $\nu$ be a p-adic measure, $f: O \rightarrow O$ a continuous map. We define

$$
\int_{O} f(x) d \nu(x):=\lim _{\leftarrow} \sum_{\bar{a} \in O / P^{m}} \nu\left(f^{-1}\left(a+P^{m}\right)\right) f(a) \in \lim _{\leftarrow} O / P^{m}=O .
$$

(iii) Let $\nu$ be a $\mathbb{Z}$-valued measure, $f:\left(O-P^{e}\right) \rightarrow\left(O-P^{e}\right)$ a continuous map $(e \in \mathbb{N})$. We define

$$
f_{O-P^{e}} f(x) d \nu(x):=\lim _{\leftarrow} \prod_{\bar{a} \in\left(O-P^{e}\right) /\left(1+P^{m}\right)} f(a)^{\nu\left(f^{-1}\left(a+P^{m}\right)\right)} \in O .
$$


We recall the setting in $[\mathrm{Da}$. Let $F$ be a totally real field of degree $n, \mathfrak{f}$ an integral ideal of $F, \mathfrak{p}:=\mathfrak{p}_{\text {id }}$ the prime ideal corresponding to the $p$-adic topology on $F$ induced by id: $F \hookrightarrow \mathbb{C}_{p}$. We assume that $\mathfrak{p} \nmid \mathfrak{f}$.

Definition 10 ([Da, Definitions 3.8, 3.9]). Let $\eta$ be a prime ideal of $F$.

(i) We say $\eta$ is good for a cone $C\left(v_{1}, \ldots, v_{r}\right)$ if $v_{i} \in \mathcal{O}_{F}-\eta$ and if $N \eta$ is a rational prime (i.e., the residue degree $=1$ ).

(ii) We say $\eta$ is good for a Shintani set $D$ if it can be expressed as a finite disjoint union of cones for which $\eta$ is good.

Definition 11 ([Da, Definitions 3.13, 3.16, Conjecture 3.21]). We take an element $\pi \in$ $\mathcal{O}_{F,+}$, a prime ideal $\eta$, a Shintani domain $\mathcal{D}_{\mathfrak{f}} \bmod E_{\mathfrak{f},+}$ satisfying the following conditions.

(i) Let $e$ be the order of $\mathfrak{p}$ in $C_{\mathfrak{f}}$. We fix a generator $\pi \in \mathfrak{p}^{e}$ satisfying $\pi \in \mathcal{O}_{F,+}$, $\pi \equiv 1 \bmod \mathfrak{f}$.

(ii) $N \eta \geq n+2$ and $(N \eta, \mathfrak{f p})=1$.

(iii) The residue degree of $\eta=1$ and the ramification degree of $\eta \leq N \eta-2$.

(iv) $\eta$ is "simultaneously" good for $\mathcal{D}_{\mathfrak{f}}, \pi^{-1} \mathcal{D}_{\mathfrak{f}}$ in the following sense: There exist vectors $\boldsymbol{v}_{j} \in\left(\mathcal{O}_{F,+}-\eta\right)^{r(j)}$, units $\epsilon_{j} \in E_{\mathfrak{f},+}\left(j \in J^{\prime},\left|J^{\prime}\right|<\infty\right)$ satisfying

$$
\mathcal{D}_{\mathfrak{f}}=\coprod_{j \in J^{\prime}} C\left(\boldsymbol{v}_{j}\right), \quad \pi^{-1} \mathcal{D}_{\mathfrak{f}}=\coprod_{j \in J^{\prime}} \epsilon_{j} C\left(\boldsymbol{v}_{j}\right) .
$$

Remark 2. Dasgupta $[\mathrm{Da}$ took a suitable set $T$ of prime ideals instead of one prime ideal $\eta$. In this article, we assume that $|T|=1$ for simplicity.

We denote by $F_{\mathfrak{p}}, \mathcal{O}_{F_{\mathfrak{p}}}$ the completion of $F$ at $\mathfrak{p}$, the ring of integers of $F_{\mathfrak{p}}$ respectively. Definition 12 ([Da, Definitions 3.13, 3.17]). Let $\pi, \eta, \mathcal{D}_{\mathfrak{f}}$ be as in Definition 11, $\mathfrak{b}$ a fractional ideal of $F$ relatively prime to $\mathfrak{f p} N \eta$. We put

$$
F_{\mathfrak{f}}^{\times}:=\left\{z \in F^{\times} \mid z \equiv 1 \bmod ^{*} \mathfrak{f}\right\} .
$$

(i) For an open compact subset $\mathcal{U} \subset \mathcal{O}_{F_{\mathfrak{p}}}$, a Shintani set $D$, we put

$$
\begin{aligned}
\nu(\mathfrak{b}, D, \mathcal{U}) & :=\zeta_{N}\left(0, F_{\mathfrak{f}}^{\times} \cap \mathfrak{b}^{-1} \cap D \cap \mathcal{U}\right), \\
\nu_{\eta}(\mathfrak{b}, D, \mathcal{U}) & :=\nu(\mathfrak{b}, D, \mathcal{U})-N \eta \nu\left(\mathfrak{b} \eta^{-1}, \mathcal{D}, \mathcal{U}\right) .
\end{aligned}
$$

Here $\zeta_{N}(s, R)$ is defined in Definition 8. By [Da, Proposition 3.12] we see that

- When $\eta$ is good for $D$, we have $\nu_{\eta}(\mathfrak{b}, D, \mathcal{U}) \in \mathbb{Z}\left[N \eta^{-1}\right]$.

- When $\eta$ is good for $D$ and $N \eta \geq n+2$, we have $\nu_{\eta}(\mathfrak{b}, D, \mathcal{U}) \in \mathbb{Z}$. 
(ii) Assume that $\eta$ is good for $D$ and that $\eta \nmid p$. We define a p-adic measure $\nu_{\eta}(\mathfrak{b}, D)$ on $\mathcal{O}_{F_{\mathfrak{p}}}$ by

$$
\nu_{\eta}(\mathfrak{b}, D)(\mathcal{U}):=\nu_{\eta}(\mathfrak{b}, D, \mathcal{U}) .
$$

Under the assumption of Definition $11, \nu_{\eta}\left(\mathfrak{b}, \mathcal{D}_{\mathfrak{f}}\right)$ is a $\mathbb{Z}$-valued measure.

(iii) For $\tau \in \operatorname{Gal}\left(H_{\mathfrak{f}} / F\right)$, we put

$$
\begin{aligned}
\zeta_{\mathfrak{f}}(s, \tau) & :=\sum_{\mathfrak{a} \subset \mathcal{O}_{F},\left(\frac{H_{\mathfrak{f}} / F}{\mathfrak{a}}\right)=\tau,(\mathfrak{a}, \mathfrak{f})=1} N \mathfrak{a}^{-s}, \\
\zeta_{\mathfrak{f}, \eta}(s, \tau) & :=\zeta_{\mathfrak{f}}(s, \tau)-N \eta^{1-s} \zeta_{\mathfrak{f}}\left(s, \tau\left(\frac{H_{\mathfrak{f}} / F}{\eta^{-1}}\right)\right) .
\end{aligned}
$$

Here $H_{\mathfrak{f}}$ denotes the narrow ray class field modulo $\mathfrak{f}$.

(iv) We define

$$
\begin{aligned}
\epsilon_{\eta}\left(\mathfrak{b}, \mathcal{D}_{\mathfrak{f}}, \pi\right) & :=\prod_{\epsilon \in E_{\mathfrak{f},+}} \epsilon^{\nu_{\eta}\left(\mathfrak{b}, \mathcal{E} \mathcal{D}_{\mathfrak{f}} \cap \pi^{-1} \mathcal{D}_{\mathfrak{f}}, \mathcal{O}_{F_{\mathfrak{p}}}\right)} \in E_{\mathfrak{f},+}, \\
u_{\eta}\left(\mathfrak{b}, \mathcal{D}_{\mathfrak{f}}\right) & :=\epsilon_{\eta}\left(\mathfrak{b}, \mathcal{D}_{\mathfrak{f}}, \pi\right) \pi^{\zeta_{\mathfrak{f}, \eta}\left(0,\left(\frac{H_{\mathfrak{f}} / F}{\mathfrak{b}}\right)\right)} \mathcal{J}_{\mathbf{O}} x d \nu_{\eta}\left(\mathfrak{b}, \mathcal{D}_{\mathfrak{f}}, x\right) \in F_{\mathfrak{p}}^{\times},
\end{aligned}
$$

where $\mathbf{O}:=\mathcal{O}_{F_{\mathfrak{p}}}-\pi \mathcal{O}_{F_{\mathfrak{p}}}$. The product in the first line is actually a finite product since $\nu_{\eta}\left(\mathfrak{b}, \epsilon \mathcal{D}_{\mathfrak{f}} \cap \pi^{-1} \mathcal{D}_{\mathfrak{f}}, \mathcal{O}_{F_{\mathfrak{p}}}\right)=0$ for all but finite $\epsilon \in E_{\mathfrak{f},+}$.

Conjecture 3 ([Da, Conjecture 3.21]). Let $\pi, \eta, \mathcal{D}_{\mathfrak{f}}$ be as in Definition 11, $H$ the fixed subfield of $H_{\mathfrak{f}}$ under $\left(\frac{H_{\mathfrak{f}} / F}{\mathfrak{p}}\right)$.

(i) Let $\tau \in \operatorname{Gal}(H / F)$. For a fractional ideal $\mathfrak{b}$ relatively prime to $\mathfrak{f p} N \eta$ satisfying $\left(\frac{H / F}{\mathfrak{b}}\right)=\tau$, we put

$$
u_{\eta}(\tau):=u_{\eta}\left(\mathfrak{b}, \mathcal{D}_{\mathfrak{f}}\right) .
$$

Then $u_{\eta}(\tau)$ depends only on $\mathfrak{f}, \tau, \eta$, not on the choices of $\mathcal{D}_{\mathfrak{f}}, \mathfrak{b}$.

(ii) For any $\tau \in \operatorname{Gal}(H / F), u_{\eta}(\tau)$ is a $\mathfrak{p}$-unit of $H$ satisfying $u_{\eta}(\tau) \equiv 1 \bmod \eta$.

(iii) For any $\tau, \tau^{\prime} \in \operatorname{Gal}(H / F)$, we have $u_{\eta}\left(\tau \tau^{\prime}\right)=u_{\eta}(\tau)^{\tau^{\prime}}$.

\section{The main results}

We keep the notation in the previous sections: Let $F$ be a totally real field of degree $n$, $H_{\mathfrak{f}}$ the narrow ray class field modulo $\mathfrak{f}$. We assume that the prime ideal $\mathfrak{p}$ corresponding to the $p$-adic topology on $F$ does not divide $\mathfrak{f}$. Let $H$ be the fixed subfield of $H_{\mathfrak{f}}$ under $\left(\frac{H_{\mathfrak{f}} / F}{\mathfrak{p}}\right)$. For $\tau \in \operatorname{Gal}(H / F)$, let $Y_{p}(\tau):=Y_{p}(\tau$, id $)$ be as in Definition 6 . For a fractional ideal $\mathfrak{b}$ relatively prime to $\mathfrak{f p} N \eta$, let $u_{\eta}\left(\mathfrak{b}, \mathcal{D}_{\mathfrak{f}}\right)$ be as in Definition 12 (iv). 
Theorem 1. We have

$$
\log _{p}\left(u_{\eta}\left(\mathfrak{b}, \mathcal{D}_{\mathfrak{f}}\right)\right)=-Y_{p}\left(\left(\frac{H / F}{\mathfrak{b}}\right)\right)+N \eta Y_{p}\left(\left(\frac{H / F}{\mathfrak{b} \eta^{-1}}\right)\right) .
$$

Corollary 1. Conjecture 2 implies

$$
u_{\eta}\left(\mathfrak{b}, \mathcal{D}_{\mathfrak{f}}\right)^{h_{H}} \equiv \prod_{\sigma \in \operatorname{Gal}(H / F)} \alpha_{H}^{\zeta_{\mathfrak{f}, \eta}\left(0, \sigma^{-1}\right)\left(\frac{H / F}{\mathfrak{b}}\right) \sigma} \bmod \operatorname{ker} \log _{p},
$$

where $\mathfrak{p}_{H}, h_{H}, \alpha_{H}$ are the prime ideal of $H$ corresponding to the p-adic topology on $H$, the class number of $H$, a generator of $\mathfrak{p}_{H}^{h_{H}}$.

We prepare some Lemmas in order prove this Theorem.

Lemma 2 ([CN2, Théorème 13]). Let $\boldsymbol{v} \in F_{+}^{r}, z \in F_{+}, \boldsymbol{\xi}=\left(\xi_{1}, \ldots, \xi_{r}\right)$ with $\xi_{i}$ roots of unity, $\neq 1$. For $k \in \mathbb{Z}_{\geq 0}$, we have

$$
\begin{aligned}
\zeta_{N}(-k, \boldsymbol{v}, z, \boldsymbol{\xi}) & =\sum_{\boldsymbol{m}=\left(m_{1}, \ldots, m_{r}\right) \in \mathbb{N}^{r}} \frac{\sum_{\boldsymbol{l}=\left(l_{1}, \ldots, l_{r}\right), 1 \leq l_{i} \leq m_{i}\left\{\begin{array}{c}
\boldsymbol{m} \\
\boldsymbol{l}
\end{array}\right\} N\left(z-\boldsymbol{l}^{t} \boldsymbol{v}\right)^{k}}^{(\mathbf{1}-\boldsymbol{\xi})^{\boldsymbol{m}}}}{(-k, \boldsymbol{v}, z, \boldsymbol{\xi})}=\sum_{\boldsymbol{m}=\left(m_{1}, \ldots, m_{r}\right) \in \mathbb{N}^{r}} \frac{\sum_{\boldsymbol{l}=\left(l_{1}, \ldots, l_{r}\right), 1 \leq l_{i} \leq m_{i}\left\{\begin{array}{c}
\boldsymbol{m} \\
\boldsymbol{l}
\end{array}\right\}\left(z-\boldsymbol{l}^{t} \boldsymbol{v}\right)^{k}}^{(\mathbf{1}-\boldsymbol{\xi})^{\boldsymbol{m}}}}{}
\end{aligned}
$$

Here we put $(\mathbf{1}-\boldsymbol{\xi})^{\boldsymbol{m}}:=\prod_{i=1}^{r}\left(1-\xi_{i}\right)^{m_{i}},\left\{\begin{array}{c}\boldsymbol{m} \\ \boldsymbol{l}\end{array}\right\}:=\prod_{i=1}^{r}\left((-1)^{l_{i}-1}\left(\begin{array}{c}m_{i}-1 \\ l_{i}-1\end{array}\right)\right)$ with the binomial coefficient $\left(\begin{array}{c}m_{i}-1 \\ l_{i}-1\end{array}\right)$. The sum over $\boldsymbol{m}$ is actually a finite sum since we have $\sum_{\boldsymbol{l}}\left\{\begin{array}{c}\boldsymbol{m} \\ \boldsymbol{l}\end{array}\right\} N(z-$ $\left.\boldsymbol{l}^{t} \boldsymbol{v}\right)^{k}=\sum_{\boldsymbol{l}}\left\{\begin{array}{c}\boldsymbol{m} \\ \boldsymbol{l}\end{array}\right\}\left(z-\boldsymbol{l}^{t} \boldsymbol{v}\right)^{k}=0$ if $m_{i}$ is large enough.

Lemma 3. Let $\nu_{\eta}(\mathfrak{b}, D, \mathcal{U})$ be as in Definition 12 -(i). Assume that $\eta$ is good for $D$. Then we have

$$
\nu_{\eta}(\mathfrak{b}, D, \mathcal{U})=\zeta\left(0, F_{\mathfrak{f}}^{\times} \cap \mathfrak{b}^{-1} \cap D \cap \mathcal{U}\right)-N \eta \zeta\left(0, F_{\mathfrak{f}}^{\times} \cap \mathfrak{b}^{-1} \eta \cap D \cap \mathcal{U}\right) .
$$

Proof. It is enough to show the statement when

- $D$ is a cone $C(\boldsymbol{v})$ with $\boldsymbol{v}=\left(v_{1}, \ldots, v_{r}\right), v_{i} \in \mathcal{O}_{F}-\eta$.

- $\mathcal{U}$ is of the form $a+\mathfrak{p}^{m} \mathcal{O}_{F_{\mathfrak{p}}}\left(m \in \mathbb{N}, a \in \mathcal{O}_{F_{\mathfrak{p}}}\right)$.

Put $R:=F_{\mathfrak{f}}^{\times} \cap \mathfrak{b}^{-1} \cap C(\boldsymbol{v}) \cap\left(a+\mathfrak{p}^{m} \mathcal{O}_{F_{\mathfrak{p}}}\right)$. By definition we have

$$
\begin{aligned}
\nu_{\eta}\left(\mathfrak{b}, C(\boldsymbol{v}), a+\mathfrak{p}^{m} \mathcal{O}_{F_{\mathfrak{p}}}\right) & =\zeta_{N}(0, R)-N \eta \zeta_{N}\left(0,\left\{z \in R \mid \operatorname{ord}_{\eta} z>0\right\}\right) \\
& =\left[\sum_{z \in R} N z^{-s}-N \eta \sum_{z \in R, \operatorname{ord}_{\eta} z>0} N z^{-s}\right]_{s=0} .
\end{aligned}
$$


Let $L$ be a positive integer satisfying $L \in \mathfrak{f p}^{m} \mathfrak{b}^{-1},(\eta, L)=1$. Then we have

$$
\begin{aligned}
\sum_{z \in R} N z^{-s} & =\sum_{\boldsymbol{x} \in R_{a}} \sum_{\boldsymbol{m} \in \mathbb{Z}_{\geq 0}^{r}} N\left((\boldsymbol{x}+\boldsymbol{m})^{t}(L \boldsymbol{v})\right)^{-s}, \\
R_{a} & :=\left\{\boldsymbol{x} \in(\mathbb{Q} \cap(0,1])^{r} \mid \boldsymbol{x}^{t}(L \boldsymbol{v}) \in F_{\mathfrak{f}}^{\times} \cap \mathfrak{b}^{-1} \cap\left(a+\mathfrak{p}^{m} \mathcal{O}_{F_{\mathfrak{p}}}\right)\right\} .
\end{aligned}
$$

Since $N \eta$ is a rational prime, the following homomorphism is a surjection.

$$
\mathbb{Z} \rightarrow \mathbb{Z} / N \eta \cong \mathcal{O}_{F} / \eta \cong \mathcal{O}_{F(\eta)} / \eta \mathcal{O}_{F(\eta)}
$$

Here we denote the localization of $\mathcal{O}_{F}$ at $\eta$ by $\mathcal{O}_{F(\eta)}$. Hence for each $\boldsymbol{x} \in R_{a}$, there exists an integer $n_{\boldsymbol{x}}$ satisfying $\boldsymbol{x}^{t} \boldsymbol{v} \equiv n_{\boldsymbol{x}} \bmod \eta \mathcal{O}_{F(\eta)}$. Similarly we take $n_{i}$ satisfying $L v_{i} \equiv n_{i} \bmod \eta \mathcal{O}_{F(\eta)}$ and put $\boldsymbol{n}_{L \boldsymbol{v}}:=\left(n_{1}, \ldots, n_{r}\right)$. Then the following are equivalent:

$$
\operatorname{ord}_{\eta}\left((\boldsymbol{x}+\boldsymbol{m})^{t}(L \boldsymbol{v})\right)>0 \Leftrightarrow n_{\boldsymbol{x}}+\boldsymbol{m}^{t} \boldsymbol{n}_{L \boldsymbol{v}} \equiv 0 \bmod N \eta .
$$

Let $\zeta$ be a primitive $N \eta$ th root of unity. We put $\xi_{\boldsymbol{x}}:=\zeta^{n_{\boldsymbol{x}}}, \xi_{i}:=\zeta^{n_{i}}, \boldsymbol{\xi}_{L \boldsymbol{v}}:=\left(\xi_{1}, \ldots, \xi_{r}\right)$. Note that $\xi_{i} \neq 1$ for any $i$. Then we have

$$
\sum_{\lambda=1}^{N \eta-1}\left(\xi_{\boldsymbol{x}} \boldsymbol{\xi}_{L \boldsymbol{v}}^{\boldsymbol{m}}\right)^{\lambda}= \begin{cases}-1 & \left(\operatorname{ord}_{\eta}\left((\boldsymbol{x}+\boldsymbol{m})^{t}(L \boldsymbol{v})\right)=0\right) \\ N \eta-1 & \left(\operatorname{ord}_{\eta}\left((\boldsymbol{x}+\boldsymbol{m})^{t}(L \boldsymbol{v})\right)>0\right)\end{cases}
$$

Here we put $\boldsymbol{\xi}_{L \boldsymbol{v}}^{m}:=\prod_{i=1}^{r} \xi_{i}^{m_{i}}$. It follows that

$$
\begin{aligned}
\sum_{\boldsymbol{x} \in R_{a}} \sum_{\lambda=1}^{N \eta-1} \xi_{\boldsymbol{x}}^{\lambda} \zeta_{N}\left(s, L \boldsymbol{v}, \boldsymbol{x}, \boldsymbol{\xi}_{L \boldsymbol{v}}^{\lambda}\right) & =\sum_{\boldsymbol{x} \in R_{a}} \sum_{\boldsymbol{m} \in \mathbb{Z}_{\geq 0}^{r}} \sum_{\lambda=1}^{N \eta-1}\left(\xi_{\boldsymbol{x}} \boldsymbol{\xi}_{L \boldsymbol{v}}^{\boldsymbol{m}}\right)^{\lambda} N\left((\boldsymbol{x}+\boldsymbol{m})^{t}(L \boldsymbol{v})\right)^{-s} \\
& =-\sum_{z \in R} N z^{-s}+N \eta \sum_{z \in Z, \operatorname{ord}_{\eta} z>0} N z^{-s} .
\end{aligned}
$$

Similarly we obtain

$$
\begin{aligned}
\sum_{\boldsymbol{x} \in R_{a}} \sum_{\lambda=1}^{N \eta-1} \xi_{\boldsymbol{x}}^{\lambda} \zeta\left(s, L \boldsymbol{v}, \boldsymbol{x}, \boldsymbol{\xi}_{L \boldsymbol{v}}^{\lambda}\right) & =-\sum_{z \in R} z^{-s}+N \eta \sum_{z \in Z, \operatorname{ord}_{\eta} z>0} z^{-s} \\
& =\zeta(s, R)-N \eta \zeta\left(s,\left\{z \in R \mid \operatorname{ord}_{\eta} z>0\right\}\right) .
\end{aligned}
$$

By Lemma 2, we have for $\boldsymbol{x} \in R_{a}$

$$
\zeta_{N}\left(0, L \boldsymbol{v}, \boldsymbol{x}, \boldsymbol{\xi}_{L \boldsymbol{v}}^{\lambda}\right)=\zeta\left(0, L \boldsymbol{v}, \boldsymbol{x}, \boldsymbol{\xi}_{L \boldsymbol{v}}^{\lambda}\right)
$$

Then the assertion follows from (6), (7), (8), (9).

Dasgupta's $p$-adic integration $\int d \nu_{\eta}\left(\mathfrak{b}, \mathcal{D}_{\mathfrak{f}}, x\right)$ is originally associated with special values of multiple zeta functions "with the norm" $\zeta_{N}(\cdots)$. By the above Lemma, we can rewrite it in terms of special values of multiple zeta functions "without the norm" $\zeta(\cdots)$. This observation is one of the main discoveries in this paper. 
Lemma 4. Let $\nu_{\eta}(\mathfrak{b}, D, \mathcal{U}), \mathbf{O}=\mathcal{O}_{F_{\mathfrak{p}}}-\pi \mathcal{O}_{F_{\mathfrak{p}}}$ be as in Definition 12. Assume that $\eta$ is good for $D$. Then we have for $k \in \mathbb{Z}_{\geq 0}, s \in \mathbb{Z}_{p}$

$$
\begin{gathered}
\int_{\mathbf{O}} x^{k} d \nu_{\eta}(\mathfrak{b}, D, x)=\zeta\left(-k, F_{\mathfrak{f}}^{\times} \cap \mathfrak{b}^{-1} \cap D \cap \mathbf{O}\right)-N \eta \zeta\left(-k, F_{\mathfrak{f}}^{\times} \cap \mathfrak{b}^{-1} \eta \cap D \cap \mathbf{O}\right), \\
\int_{\mathbf{O}}\langle x\rangle^{-s} d \nu_{\eta}(\mathfrak{b}, D, x)=\zeta_{p}\left(s, F_{\mathfrak{f}}^{\times} \cap \mathfrak{b}^{-1} \cap D \cap \mathbf{O}\right)-N \eta \zeta_{p}\left(s, F_{\mathfrak{f}}^{\times} \cap \mathfrak{b}^{-1} \eta \cap D \cap \mathbf{O}\right) .
\end{gathered}
$$

Here we put $\langle x\rangle:=p^{-\operatorname{ord}_{p} x} \theta_{p}(x)^{-1} x$ by using $\operatorname{ord}_{p}, \theta_{p}$ in (2).

Proof. It is enough to show the statement when $D=C(\boldsymbol{v})$ with $\boldsymbol{v}=\left(v_{1}, \ldots, v_{r}\right), v_{i} \in$ $\mathcal{O}_{F}-\eta$. By definition we can write

$$
\int_{\mathbf{O}} x^{k} d \nu_{\eta}(\mathfrak{b}, C(\boldsymbol{v}), x)=\lim _{\leftarrow} \sum_{\bar{a} \in \mathbf{O} /\left(1+\mathfrak{p}^{m} \mathcal{O}_{F_{\mathfrak{p}}}\right)} a^{k} \nu_{\eta}\left(\mathfrak{b}, C(\boldsymbol{v}), a\left(1+\mathfrak{p}^{m} \mathcal{O}_{F_{\mathfrak{p}}}\right)\right) .
$$

By Lemmas 2, 3, we have

$$
a^{k} \nu_{\eta}\left(\mathfrak{b}, C(\boldsymbol{v}), a\left(1+\mathfrak{p}^{m} \mathcal{O}_{F_{\mathfrak{p}}}\right)\right)=-\sum_{\boldsymbol{m} \in \mathbb{N}^{r}} \sum_{\lambda=1}^{N \eta-1} \sum_{\boldsymbol{x} \in R_{a}} \frac{\xi_{\boldsymbol{x}}^{\lambda} \sum_{1 \leq l_{i} \leq m_{i}}\left\{\begin{array}{c}
\boldsymbol{m} \\
\boldsymbol{l}
\end{array}\right\} a^{k}}{\left(\mathbf{1}-\boldsymbol{\xi}_{L \boldsymbol{v}}^{\lambda}\right)^{\boldsymbol{m}}}
$$

where $L, R_{a}, \xi_{\boldsymbol{x}}, \boldsymbol{\xi}_{L \boldsymbol{v}}$ are as in the proof of Lemma 3. On the other hand, by Lemma 2 again, we obtain

$$
\begin{gathered}
\zeta\left(-k, F_{\mathfrak{f}}^{\times} \cap \mathfrak{b}^{-1} \cap C(\boldsymbol{v}) \cap \mathbf{O}\right)-N \eta \zeta\left(-k, F_{\mathfrak{f}}^{\times} \cap \mathfrak{b}^{-1} \eta \cap C(\boldsymbol{v}) \cap \mathbf{O}\right) \\
=-\sum_{\bar{a} \in \mathbf{O} /\left(1+\mathfrak{p}^{m} \mathcal{O}_{F_{\mathfrak{p}}}\right)} \sum_{\boldsymbol{x} \in R_{a}} \sum_{\boldsymbol{m} \in \mathbb{N}^{r}} \sum_{\lambda=1}^{N \eta-1} \frac{\xi_{\boldsymbol{x}}^{\lambda} \sum_{1 \leq l_{i} \leq m_{i}}\left\{\begin{array}{c}
\boldsymbol{m} \\
\boldsymbol{l}
\end{array}\right\}\left((\boldsymbol{x}-\boldsymbol{l})^{t}(L \boldsymbol{v})\right)^{k}}{\left(\mathbf{1}-\boldsymbol{\xi}_{L \boldsymbol{v}}^{\lambda}\right)^{\boldsymbol{m}}} .
\end{gathered}
$$

By definition, we see that $L \in \mathfrak{p}^{m}, \boldsymbol{x}^{t}(L \boldsymbol{v}) \equiv a \bmod \mathfrak{p}^{m} \mathcal{O}_{F_{\mathfrak{p}}}$ for $\boldsymbol{x} \in R_{a}$. It follows that

$$
a^{k} \equiv\left((\boldsymbol{x}-\boldsymbol{l})^{t}(L \boldsymbol{v})\right)^{k} \bmod \mathfrak{p}^{m} \mathcal{O}_{F_{\mathfrak{p}}} \quad\left(\boldsymbol{x} \in R_{a}\right),
$$

Hence the first assertion is clear. The second assertion follows from the $p$-adic interpolation property (4).

Proof of Theorem 1. For a fractional ideal $\mathfrak{b}$, a Shintani set $D$, an open compact subset $\mathcal{U} \subset \mathcal{O}_{F_{\mathrm{p}}}$, and for $*=\emptyset, p$, we put

$$
\begin{aligned}
L \Gamma_{*}(\mathfrak{b}, D, \mathcal{U}) & :=L \Gamma_{*}\left(F_{\mathfrak{f}}^{\times} \cap \mathfrak{b}^{-1} \cap D \cap \mathcal{U}\right), \\
L \Gamma_{\eta, *}(\mathfrak{b}, D, \mathcal{U}) & :=L \Gamma_{*}(\mathfrak{b}, D, \mathcal{U})-N \eta L \Gamma_{*}\left(\mathfrak{b} \eta^{-1}, D, \mathcal{U}\right)
\end{aligned}
$$

whenever each function is well-defined. It suffices to show the following three equalities:

$$
\begin{aligned}
\log _{p}\left(\epsilon_{\eta}\left(\mathfrak{b}, \mathcal{D}_{\mathfrak{f}}, \pi\right) \pi^{\zeta_{\mathfrak{f}, \eta}\left(0,\left(\frac{H_{\mathfrak{f}} / F}{\mathfrak{b}}\right)\right)}\right) & =\left[L \Gamma_{\eta}\left(\mathfrak{b}, \mathcal{D}_{\mathfrak{f}}, \mathbf{O}\right)\right]_{p}, \\
\log _{p}\left(\mathcal{F}_{\mathbf{O}} x d \nu_{\eta}\left(\mathfrak{b}, \mathcal{D}_{\mathfrak{f}}, x\right)\right) & =-L \Gamma_{\eta, p}\left(\mathfrak{b}, \mathcal{D}_{\mathfrak{f}}, \mathbf{O}\right), \\
L \Gamma_{p}\left(\mathfrak{b}, \mathcal{D}_{\mathfrak{f}}, \mathbf{O}\right)-\left[L \Gamma\left(\mathfrak{b}, \mathcal{D}_{\mathfrak{f}}, \mathbf{O}\right)\right]_{p} & =Y_{p}\left(\left(\frac{H / F}{\mathfrak{b}}\right)\right) .
\end{aligned}
$$


Let $\boldsymbol{v}_{j}, \epsilon_{j}\left(j \in J^{\prime}\right)$ be as in Definition 11 -(iv). Since $\mathcal{D}_{\mathfrak{f}}, \pi^{-1} \mathcal{D}_{\mathfrak{f}}$ are fundamental domains of $F \otimes \mathbb{R}_{+} / E_{\mathfrak{f},+}$, we see that

$$
\epsilon \mathcal{D}_{\mathfrak{f}} \cap \pi^{-1} \mathcal{D}_{\mathfrak{f}}=\coprod_{j \in J^{\prime}, \epsilon_{j}=\epsilon} \epsilon_{j} C\left(\boldsymbol{v}_{j}\right) \quad\left(\epsilon \in E_{\mathfrak{f},+}\right)
$$

Namely we have

$$
\epsilon_{\eta}\left(\mathfrak{b}, \mathcal{D}_{\mathfrak{f}}, \pi\right)=\prod_{j \in J^{\prime}} \epsilon_{j}^{\nu_{\eta}\left(\mathfrak{b}, \epsilon_{j} C\left(\boldsymbol{v}_{j}\right), \mathcal{O}_{F_{\mathfrak{p}}}\right)}
$$

By Lemma 3, we can write

$$
\begin{aligned}
& \nu_{\eta}\left(\mathfrak{b}, \epsilon_{j} C\left(\boldsymbol{v}_{j}\right), \mathcal{O}_{F_{\mathfrak{p}}}\right) \\
& =\zeta\left(0, F_{\mathfrak{f}}^{\times} \cap \mathfrak{b}^{-1} \cap \epsilon_{j} C\left(\boldsymbol{v}_{j}\right) \cap \mathcal{O}_{F_{\mathfrak{p}}}\right)-N \eta \zeta\left(0, F_{\mathfrak{f}}^{\times} \cap \mathfrak{b}^{-1} \eta \cap \epsilon_{j} C\left(\boldsymbol{v}_{j}\right) \cap \mathcal{O}_{F_{\mathfrak{p}}}\right) .
\end{aligned}
$$

Therefore by Proposition 1 1 (i) we obtain

$$
\begin{aligned}
\log _{p}\left(\epsilon_{\eta}\left(\mathfrak{b}, \mathcal{D}_{\mathfrak{f}}, \pi\right)\right) & =\left[\sum_{j \in J^{\prime}} L \Gamma_{\eta}\left(\mathfrak{b}, C\left(\boldsymbol{v}_{j}\right), \mathcal{O}_{F_{\mathfrak{p}}}\right)-\sum_{j \in J^{\prime}} L \Gamma_{\eta}\left(\mathfrak{b}, \epsilon_{j} C\left(\boldsymbol{v}_{j}\right), \mathcal{O}_{F_{\mathfrak{p}}}\right)\right]_{p} \\
& =\left[L \Gamma_{\eta}\left(\mathfrak{b}, \mathcal{D}_{\mathfrak{f}}, \mathcal{O}_{F_{\mathfrak{p}}}\right)-L \Gamma_{\eta}\left(\mathfrak{b}, \pi^{-1} \mathcal{D}_{\mathfrak{f}}, \mathcal{O}_{F_{\mathfrak{p}}}\right)\right]_{p} .
\end{aligned}
$$

We easily see that

$$
\begin{aligned}
\zeta_{\mathfrak{f}, \eta}\left(0,\left(\frac{H_{\mathfrak{f}} / F}{\mathfrak{b}}\right)\right) & =\nu_{\eta}\left(\mathfrak{b}, \mathcal{D}_{\mathfrak{f}}, \mathcal{O}_{F_{\mathfrak{p}}}\right) \\
\pi\left(F_{\mathfrak{f}}^{\times} \cap \mathfrak{b}^{-1} \cap \pi^{-1} \mathcal{D}_{\mathfrak{f}} \cap \mathcal{O}_{F_{\mathfrak{p}}}\right) & =F_{\mathfrak{f}}^{\times} \cap \pi \mathfrak{b}^{-1} \cap \mathcal{D}_{\mathfrak{f}} \cap \pi \mathcal{O}_{F_{\mathfrak{p}}} .
\end{aligned}
$$

Hence, by Proposition 1. (i) again, we get

$$
\log _{p}\left(\pi^{\zeta_{\mathfrak{f}, \eta}\left(0,\left(\frac{H_{\mathfrak{f}} / F}{\mathfrak{b}}\right)\right)}\right)=\left[L \Gamma_{\eta}\left(\mathfrak{b}, \pi^{-1} \mathcal{D}_{\mathfrak{f}}, \mathcal{O}_{F_{\mathfrak{p}}}\right)-L \Gamma_{\eta}\left(\pi^{-1} \mathfrak{b}, \mathcal{D}_{\mathfrak{f}}, \pi \mathcal{O}_{F_{\mathfrak{p}}}\right)\right]_{p}
$$

Since $\left(F_{\mathfrak{f}}^{\times} \cap \mathfrak{b}^{-1} \cap \mathcal{D}_{\mathfrak{f}} \cap \mathbf{O}\right) \coprod\left(F_{\mathfrak{f}}^{\times} \cap \pi \mathfrak{b}^{-1} \cap \mathcal{D}_{\mathfrak{f}} \cap \pi \mathcal{O}_{F_{\mathfrak{p}}}\right)=F_{\mathfrak{f}}^{\times} \cap \mathfrak{b}^{-1} \cap \mathcal{D}_{\mathfrak{f}} \cap \mathcal{O}_{F_{\mathfrak{p}}}$, we have

$$
L \Gamma_{\eta}\left(\mathfrak{b}, \mathcal{D}_{\mathfrak{f}}, \mathbf{O}\right)=L \Gamma_{\eta}\left(\mathfrak{b}, \mathcal{D}_{\mathfrak{f}}, \mathcal{O}_{F_{\mathfrak{p}}}\right)-L \Gamma_{\eta}\left(\pi^{-1} \mathfrak{b}, \mathcal{D}_{\mathfrak{f}}, \pi \mathcal{O}_{F_{\mathfrak{p}}}\right)
$$

Then the assertion (11) follows from (14), (15), (16).

Next, differentiating (10) at $s=0$, we obtain

$$
-\int_{\mathbf{O}} \log _{p} x d \nu_{\eta}\left(\mathfrak{b}, \mathcal{D}_{\mathfrak{f}}, x\right)=L \Gamma_{\eta, p}\left(\mathfrak{b}, \mathcal{D}_{\mathfrak{f}}, \mathbf{O}\right)
$$

By definition, we have $\log _{p}\left(\mathscr{f}_{\mathbf{O}} x d \nu_{\eta}\left(\mathfrak{b}, \mathcal{D}_{\mathfrak{f}}, x\right)\right)=\int_{\mathbf{O}} \log _{p} x d \nu_{\eta}\left(\mathfrak{b}, \mathcal{D}_{\mathfrak{f}}, x\right)$. Hence the assertion 12 is clear.

Finally we prove $(13)$. Let $D$ be a Shintani domain $\bmod E_{+}$. For each $c \in C_{\mathfrak{f p}}$, we take an integral ideal $\mathfrak{a}_{c}$ satisfying $\mathfrak{a}_{c} \mathfrak{f} \in \pi(c)$, and put $R_{c}:=\left\{z \in D \mid \mathcal{O}_{F} \supset z \mathfrak{a}_{c} \mathfrak{f} \mathfrak{p} \in c\right\}$. By (5) we can write

$$
Y_{p}\left(\left(\frac{H / F}{\mathfrak{b}}\right)\right)=\sum_{c \in C_{\mathfrak{f p}},\left.\operatorname{Art}(\bar{c})\right|_{H}=\left(\frac{H / F}{\mathfrak{b}}\right)} L \Gamma_{p}\left(R_{c}\right)-\left[\sum_{c \in C_{\mathfrak{f p}},} \sum_{\left.\operatorname{Art}(\bar{c})\right|_{H}=\left(\frac{H / F}{\mathfrak{b}}\right)} L \Gamma\left(R_{c}\right)\right]_{p} .
$$


Since $H$ is the fixed subfield under $\left(\frac{H_{\mathfrak{f}} / F}{\mathfrak{p}}\right)$, we may replace

$$
\sum_{c \in C_{\mathfrak{f p}},\left.\operatorname{Art}(\bar{c})\right|_{H}=\left(\frac{H / F}{\mathfrak{b}}\right)} \cdots=\sum_{k=0}^{e-1} \sum_{c \in C_{\mathfrak{f p}}, \bar{c}=[\mathfrak{b p}-k]} \cdots,
$$

where $\bar{c}$ denotes the image under $C_{\mathfrak{f p}} \rightarrow C_{\mathfrak{f}}$, [a $]$ denotes the ideal class in $C_{\mathfrak{f}}$ of a fractional ideal $\mathfrak{a}$. On the other hand, we can write for $*=\emptyset, p$

$$
L \Gamma_{*}\left(\mathfrak{b}, \mathcal{D}_{\mathfrak{f}}, \mathbf{O}\right)=\sum_{k=0}^{e-1} L \Gamma_{*}\left(\mathfrak{b}, \mathcal{D}_{\mathfrak{f}}, \mathfrak{p}^{k} \mathcal{O}_{F_{\mathfrak{p}}}^{\times}\right)
$$

Therefore it suffices to show that we have for each $k$

$$
\begin{aligned}
& \left(\sum_{c \in C_{\mathfrak{f p}}, \bar{c}=[\mathfrak{b p}-k]} L \Gamma_{p}\left(R_{c}\right)\right)-L \Gamma_{p}\left(\mathfrak{b}, \mathcal{D}_{\mathfrak{f}}, \mathfrak{p}^{k} \mathcal{O}_{F_{\mathfrak{p}}}^{\times}\right) \\
& =\left[\left(\sum_{c \in C_{\mathfrak{f p}}, \bar{c}=[\mathfrak{b p}-k]} L \Gamma\left(R_{c}\right)\right)-L \Gamma\left(\mathfrak{b}, \mathcal{D}_{\mathfrak{f}}, \mathfrak{p}^{k} \mathcal{O}_{F_{\mathfrak{p}}}^{\times}\right)\right]_{p} .
\end{aligned}
$$

We fix $k$. Whenever $\bar{c}=\left[\mathfrak{b} \mathfrak{p}^{-k}\right], \pi(c) \in C_{(1)}$ is constant, so we may put $\mathfrak{a}_{c}$ to be a fixed integral ideal $\mathfrak{a}_{0}$. Then we have

$$
\coprod_{c \in C_{\mathfrak{f} p}, \bar{c}=\left[\mathfrak{b p}^{-k}\right]} R_{c}=\left\{z \in\left(\mathfrak{a}_{0} \mathfrak{f p}\right)^{-1} \cap D \mid\left(z \mathfrak{a}_{0} \mathfrak{f} \mathfrak{p}, \mathfrak{f p}\right)=1, \quad\left[z \mathfrak{a}_{0} \mathfrak{f} \mathfrak{p}\right]=\left[\mathfrak{b} \mathfrak{p}^{-k}\right] \text { in } C_{\mathfrak{f}}\right\}
$$

Let $\alpha_{0} \in F_{+}$be a generator of the principal ideal $\left(\mathfrak{a}_{0} \mathfrak{f} \mathfrak{p}\right)\left(\mathfrak{b} \mathfrak{p}^{-k}\right)^{-1}$. Then the following are equivalent:

$$
\left[z \mathfrak{a}_{0} \mathfrak{f} \mathfrak{p}\right]=\left[\mathfrak{b}^{-k}\right] \Leftrightarrow\left[\left(z \alpha_{0}\right)\right]=[(1)] \Leftrightarrow \exists \epsilon \in E_{+} \text {s.t. } z \epsilon \alpha_{0} \equiv 1 \bmod \mathfrak{f} .
$$

Hence, taking a representative set $E_{0}$ of $E_{+} / E_{\mathfrak{f},+}$, we can write

$$
\begin{aligned}
& \left\{z \in\left(\mathfrak{a}_{0} \mathfrak{f} \mathfrak{p}\right)^{-1} \cap D \mid\left(z \mathfrak{a}_{0} \mathfrak{f} \mathfrak{p}, \mathfrak{f p}\right)=1,\left[z \mathfrak{a}_{0} \mathfrak{f} \mathfrak{p}\right]=\left[\mathfrak{b p}^{-k}\right] \text { in } C_{\mathfrak{f}}\right\} \\
& =\coprod_{\epsilon \in E_{0}}\left(\epsilon \alpha_{0}\right)^{-1}\left(F_{\mathfrak{f}}^{\times} \cap \mathfrak{b}^{-1} \cap \epsilon \alpha_{0} D \cap \mathfrak{p}^{k} \mathcal{O}_{F_{\mathfrak{p}}}^{\times}\right) .
\end{aligned}
$$

Namely we have for $*=\emptyset, p$

$$
\sum_{c \in C_{\mathfrak{f p}}, \bar{c}=\left[\mathfrak{b} \mathfrak{p}^{-k}\right]} L \Gamma_{*}\left(R_{c}\right)=\sum_{\epsilon \in E_{0}} L \Gamma_{*}\left(\left(\epsilon \alpha_{0}\right)^{-1}\left(F_{\mathfrak{f}}^{\times} \cap \mathfrak{b}^{-1} \cap \epsilon \alpha_{0} D \cap \mathfrak{p}^{k} \mathcal{O}_{F_{\mathfrak{p}}}^{\times}\right)\right) .
$$

On the other hand, $\mathcal{D}_{\mathfrak{f}}^{\prime}:=\coprod_{\epsilon \in E_{0}} \epsilon \alpha_{0} D$ becomes another Shintani domain $\bmod E_{\mathfrak{f},+}$, and we can write for $*=\emptyset, p$

$$
L \Gamma_{*}\left(\mathfrak{b}, \mathcal{D}_{\mathfrak{f}}^{\prime}, \mathfrak{p}^{k} \mathcal{O}_{F_{\mathfrak{p}}}^{\times}\right)=\sum_{\epsilon \in E_{0}} L \Gamma_{*}\left(F_{\mathfrak{f}}^{\times} \cap \mathfrak{b}^{-1} \cap \epsilon \alpha_{0} D \cap \mathfrak{p}^{k} \mathcal{O}_{F_{\mathfrak{p}}}^{\times}\right) .
$$


Then the assertion (17), replacing $\mathcal{D}_{\mathfrak{f}}$ with $\mathcal{D}_{\mathfrak{f}}^{\prime}$, follows from $(18),(19)$ and Proposition 1 . We conclude the proof of $(13)$ by showing that

$$
L \Gamma_{p}\left(\mathfrak{b}, \mathcal{D}_{\mathfrak{f}}, \mathfrak{p}^{k} \mathcal{O}_{F_{\mathfrak{p}}}^{\times}\right)-L \Gamma_{p}\left(\mathfrak{b}, \mathcal{D}_{\mathfrak{f}}^{\prime}, \mathfrak{p}^{k} \mathcal{O}_{F_{\mathfrak{p}}}^{\times}\right)=\left[L \Gamma\left(\mathfrak{b}, \mathcal{D}_{\mathfrak{f}}, \mathfrak{p}^{k} \mathcal{O}_{F_{\mathfrak{p}}}^{\times}\right)-L \Gamma\left(\mathfrak{b}, \mathcal{D}_{\mathfrak{f}}^{\prime}, \mathfrak{p}^{k} \mathcal{O}_{F_{\mathfrak{p}}}^{\times}\right)\right]_{p}
$$

Note that the independence on the choice of $\mathcal{D}_{\mathfrak{f}}$ is also discussed in [Da, $\left.\S 5.2\right]$ under certain conditions. Similarly to [Yo, Chap. III, Lemma 3.13], we see that there exist cones $C\left(\boldsymbol{v}_{j}\right)$ and units $u_{j} \in E_{\mathfrak{f},+}\left(j \in J^{\prime \prime}\right)$ which satisfy

$$
\mathcal{D}_{\mathfrak{f}}=\coprod_{j \in J^{\prime \prime}} C\left(\boldsymbol{v}_{j}\right), \quad \mathcal{D}_{\mathfrak{f}}^{\prime}=\coprod_{j \in J^{\prime \prime}} u_{j} C\left(\boldsymbol{v}_{j}\right) .
$$

Therefore it suffices to show that

$$
\begin{aligned}
& L \Gamma_{p}\left(\mathfrak{b}, C\left(\boldsymbol{v}_{j}\right), \mathfrak{p}^{k} \mathcal{O}_{F_{\mathfrak{p}}}^{\times}\right)-L \Gamma_{p}\left(\mathfrak{b}, u_{j} C\left(\boldsymbol{v}_{j}\right), \mathfrak{p}^{k} \mathcal{O}_{F_{\mathfrak{p}}}^{\times}\right) \\
& =\left[L \Gamma\left(\mathfrak{b}, C\left(\boldsymbol{v}_{j}\right), \mathfrak{p}^{k} \mathcal{O}_{F_{\mathfrak{p}}}^{\times}\right)-L \Gamma\left(\mathfrak{b}, u_{j} C\left(\boldsymbol{v}_{j}\right), \mathfrak{p}^{k} \mathcal{O}_{F_{\mathfrak{p}}}^{\times}\right)\right]_{p} .
\end{aligned}
$$

It follows from Proposition 1 since $F_{\mathfrak{f}}^{\times} \cap \mathfrak{b}^{-1} \cap u_{j} C\left(\boldsymbol{v}_{j}\right) \cap \mathfrak{p}^{k} \mathcal{O}_{F_{\mathfrak{p}}}^{\times}=u_{j}\left(F_{\mathfrak{f}}^{\times} \cap \mathfrak{b}^{-1} \cap C\left(\boldsymbol{v}_{j}\right) \cap\right.$ $\left.\mathfrak{p}^{k} \mathcal{O}_{F_{\mathfrak{p}}}^{\times}\right)$.

\section{References}

[CN1] P. Cassou-Noguès, Analogues p-adiques de quelques fonctions arithmétiques, Publ. Math. Bordeaux (1974-1975), 1-43.

[CN2] P. Cassou-Noguès, Valeurs aux entiers négative des fonction zêta et fonction zêta p-adiques, Inv. Math. 51 (1979), 29-59.

[Da] S. Dasgupta, Shintani zeta-functions and Gross-Stark units for totally real fields, Duke Math. J. 143 (2008), no. 2, 225-279.

[DDP] S. Dasgupta, H. Darmon, R. Pollack, Hilbert modular forms and the Gross-Stark conjecture, Ann. of Math. (2) 174 (2011), no. 1, 439-484.

[Gr] B. H. Gross, p-adic L-series at $s=0$, J. Fac. Sci. Univ. Tokyo 28 (1981), 979-994.

[Ka1] T. Kashio, On a p-adic analogue of Shintani's formula, J. Math. Kyoto Univ. 45 (2005), 99-128.

[Ka2] T. Kashio, Fermat curves and a refinement of the reciprocity law on cyclotomic units, J. Reine Angew. Math., DOI: 10.1515/crelle-2015-0081.

[Ka3] T. Kashio, On the ratios of Barnes' multiple gamma functions to the $p$-adic analogues (arXiv:1703.10411),

[KY1] T. Kashio, and H. Yoshida, On p-adic absolute CM-Periods, I, Amer. J. Math. 130 (2008), no. 6, 1629-1685. 
[KY2] T. Kashio, and H. Yoshida, On p-adic absolute CM-Periods, II, Publ. Res. Inst. Math. Sci. 45 (2009), no. 1, 187-225.

[Sh] T. Shintani, On evaluation of zeta functions of totally real algebraic number fields at non-positive integers, J. Fac. Sci. Univ. Tokyo Sect. IA Math. 23 (1976), no. 2, 393-417.

[Yo] H. Yoshida, Absolute CM-Periods, Math. Surveys Monogr. 106, Amer. Math. Soc., Providence, RI, 2003. 